\title{
II mestiere del docente. Riflessioni di Alfonso Gambardella e Salvio Vicari
}

\author{
AlFONSO GAMBARDELLA* SALVIO VICARI
}

\section{I piani del contributo del docente di management}

Il contributo che un docente di management può dare alla comunità è su tre piani: la ricerca, la didattica, il servizio. Tutti e tre egualmente importanti, ma con un peso specifico diverso in funzione di alcune circostanze.

La qualità della ricerca è elemento essenziale di un docente di ruolo: è ciò che caratterizza l'insegnamento universitario rispetto ad altri gradi d'istruzione. Senza ricerca di qualità non può esservi docente dell'università.

L'insegnamento è parte importante del mestiere di docente. L'istruzione superiore ha bisogno di persone brave nella didattica, soprattutto per i corsi undergraduate, graduate e post-experience. Alcuni docenti con spiccate capacità di ricerca svolgono egregiamente sia la didattica avanzata che quella di base. Vi sono però ottimi ricercatori meno propensi, o meno capaci, a svolgere didattica di base, così come vi sono docenti meno specializzati nella ricerca che insegnano molto bene in questi corsi. Il bilanciamento tra queste alternative lega il mestiere del docente alla strategia e al modello più generale di un Ateneo o Dipartimento. Un Ateneo o Dipartimento che vuole essere riconosciuto per le sue competenze di ricerca e per un insegnamento di alta qualità impiegherà docenti con spiccate qualità di ricerca, mentre nei suoi corsi di base potrà impiegare docenti efficaci in questo tipo di didattica anche se non ricercatori di frontiera. Altri Atenei o Dipartimenti possono scegliere di specializzarsi nella didattica, assumendo i connotati di un college universitario. In questo caso, il ruolo di docenti capaci nella didattica, ma meno versati alla ricerca, sarà preponderante.

Il servizio è importante per la vita delle università e dei loro territori. Il servizio è parte integrante dell'attività del docente e deve essere strutturato e valutato dalle università e dai dipartimenti, esistendo in questo campo una grande varietà di situazioni possibili. Il servizio non dovrebbe mai raggiungere, salvo casi eccezionali, intensità tali da compromettere seriamente la produzione scientifica e la qualità didattica dei docenti.

\footnotetext{
Ordinario di Economia e Gestione delle Imprese - Università Bocconi Milano e-mail: alfonso.gambardella@unibocconi.it

** Ordinario di Economia e Gestione delle Imprese - Università Bocconi Milano e-mail: salvatore.vicari@unibocconi.it
} 
È fondamentale ricordare che esiste un ciclo di vita del docente, in cui l'importanza dei tre ruoli cambia. Questo ciclo di vita è stato dimostrato con riferimento alle scienze fisiche e qualche anno addietro anche nelle discipline economiche (Goodwin e Sauer, 1995) ed è evidente anche con riferimento alle discipline manageriali, come si può vedere dalla Figura 1, che illustra la produttività scientifica con riferimento alle diverse coorti di età, misurata in termini di anni dal conseguimento del Ph.D.

La fase delle fondamenta scientifiche. La ricerca è fondamentale e dovrebbe assorbire la quasi totalità delle risorse cognitive e di tempo di un ricercatore nei primi anni del proprio sviluppo. Il motivo più importante è che la struttura metodologica $\mathrm{e}$ il bagaglio di conoscenze su cui costruire la propria ricerca, richiedono un periodo inziale di grande impegno. L'attività nei primi anni costituisce la base, le fondamenta, su cui poggerà la ricerca negli anni successivi. Potremmo indicare che in alcuni studi questa prima fase raggiunge il culmine circa 7 anni dopo il Phd, periodo in cui di solito si ottiene la tenure nel mondo anglosassone. In questo stadio, la didattica va fatta, ma non deve assorbire in modo eccessivo il docente, che deve mantenere spazi significatavi per l'attività di ricerca.

Fig. 1: Pubblicazioni di livello A per coorte di PhD
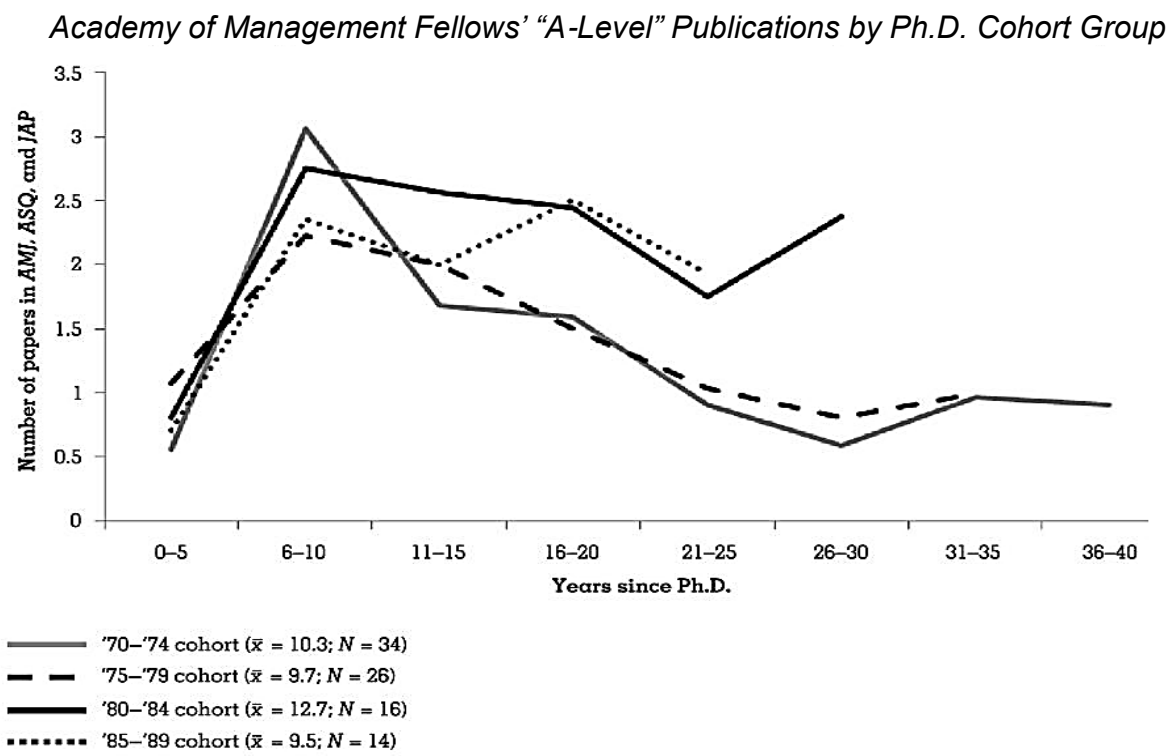

Fonte: Walsh, 2011

La fase della crescita scientifica e didattica. In una seconda fase, che potremmo collocare indicativamente fino a venti anni circa dopo il phd, la docenza progressivamente aumenta di importanza, affiancandosi all'attività di ricerca, che 
assume un connotato non solo di sviluppo individuale o in collegamento ad altri ricercatori, ma che comporta anche la guida di gruppi di ricerca e lo sviluppo delle competenze dei colleghi più giovani. In questa fase si svolge anche la ricerca applicata, dove cioè il destinatario non è solo la comunità accademica, ma coinvolge altri stakeholder. Questo tipo di ricerca rientra in parte tra le attività di servizio. In questa fase si svolgono anche altri compiti di servizio collegati sia alla ricerca sia alla didattica.

La fase della maturità scientifica. In una terza fase, quella della maturità, la didattica ed il servizio, anche in organi di governo dell'università, assumono gradualmente il peso prevalente. L'attività di ricerca si orienta anche alla diffusione scientifica, con la scrittura di monografie e di testi didattici, oltre che di opere di divulgazione in senso stretto. La ricerca originale subisce progressivamente una riduzione marcata, per una serie di motivi legati anche al fatto che l'attività di servizio assorbe molto tempo e soprattutto elevate risorse cognitive: una volta depauperato il capitale cognitivo legato alla ricerca, è molto difficile mantenere un elevato livello di produttività.

\section{La ricerca}

La ricerca consiste nel dare un contributo alla crescita della conoscenza nelle discipline manageriali alla comunità scientifica e manageriale di tutto il mondo, mediante la pubblicazione dei risultati delle propria ricerca nelle migliori sedi internazionali.

Al riguardo vi sono quattro obiezioni spesso sentite e lette, e che suonano a prima vista come del tutto legittime e di grande buon senso:

1. la comunità di riferimento non può essere solo quella internazionale, ma deve essere considerata in modo esplicito quella nazionale.

2. la nostra cultura e la nostra tradizione vanno salvaguardate.

3. il riferimento al territorio è importante e non va trascurato.

4. la vicinanza ai temi delle imprese e la rilevanza per i manager sono importanti, per cui è necessario pubblicare anche sulle riviste accademiche nazionali.

5. la pubblicazione in libri è importante, perché consente lo sviluppo di un discorso lungo e approfondito, che le riviste non consentono.

Senza avere pretese di esaustività, vorremmo dare un contributo al dibattito, cercando di dimostrare come si tratta di temi apparentemente condivisibili, ma che nascondono parecchie insidie.

\subsection{La comunità di riferimento non può essere solo quella internazionale, ma deve essere considerata in modo esplicito quella nazionale?}

Questa asserzione a noi pare priva di significato. Il motivo è che dovremmo cercare di influenzare la comunità accademica più ampia possibile, cioè quella internazionale, che naturalmente include quella nazionale. 
Se invece si considera che la comunità nazionale sia diversa da quelle di tutti gli altri paesi del mondo, allora forse siamo di fronte a un problema serio. Nessuna comunità accademica in Europa, con tradizione ed esperienza almeno pari alla nostra (ad es. tedesca, francese, spagnola), pensa di dovere avere una comunità scientifica separata da quella internazionale. Si ricordi l'importanza della scuola tedesca o di quella francese sui temi di management, che avevano riviste di grande prestigio e che hanno deciso di concentrare le proprie energie sulle riviste internazionali.

Il fatto è che la comunità manageriale si è ormai ampiamente internazionalizzata, comprendendo non più solo quella delle grandi multinazionali, ma anche delle piccole imprese ed anche delle realtà artigianali, le quali, tutte, hanno come mercato, almeno potenziale, il mondo. Non ha alcun senso pensare al management come ad una disciplina nazionale, determinata da fattori locali.

\subsection{La nostra cultura e la nostra tradizione vanno salvaguardate?}

Ciò è indubbiamente importante. Non esiste cultura che non poggi le proprie fondamenta sulla tradizione. Tuttavia la questione è cosa voglia dire salvaguardare la cultura: non certo creare steccati tra la nostra tradizione e le altre. Significa invece che dobbiamo valorizzare la nostra cultura, pubblicando sulle migliori riviste internazionali, partendo dalla nostra memoria e diffondendo le idee che affondano le radici nella nostra tradizione. Tuttavia, perché queste idee possano circolare, bisogna che siano conosciute e che abbiano un impatto sulla comunità scientifica internazionale, altrimenti divengono una sorta di archeologia culturale, la cui cura non è a nostro avviso negli obiettivi del docente di management.

Legato a questo, la valorizzazione della nostra cultura deve tenere conto di un punto importante. Fenomeni specifici del nostro contesto possono diventare modelli di riferimento della ricerca internazionale in campo manageriale se presentano due connotati che devono sussistere contemporaneamente. Il primo è che l'ambiente, i comportamenti o i meccanismi sono diversi da quanto già osservato altrove dalla letteratura. Il secondo è che siano generalizzabili a situazioni e contesti diversi dal nostro.

Per quanto riguarda il primo connotato, se situazione, comportamenti e meccanismi fossero simili a quanto già osservato altrove, la ricerca sarebbe poco interessante. Può esserlo l'applicazione di modelli teorici internazionali esistenti al sistema italiano, ma allora più che di ricerca parliamo di un modo per studiare dei fenomeni che interessano, tra gli altri, anche il nostro Paese, più utile alla divulgazione, alla consulenza, alle politiche che non alla ricerca. Il secondo connotato è altrettanto importante. La specificità italiana è tanto più interessante per la ricerca, quanto più informa e fa comprendere anche realtà diverse dalla nostra.

Ad esempio, perché la ricerca sui distretti industriali italiani ha avuto un certo impatto internazionale? Anzitutto, è un fenomeno non completamente spiegabile con le teorie tradizionali. Ad esempio, la teoria dei costi di transazione suggerisce che imprese iper-specializzate s'integrano in aziende più grandi perché è più forte il rischio di opportunismo nelle negoziazioni di mercato. Nei distretti, gli imprenditori 
sono legati da un milieu culturale: le imprese restano separate perché gli accordi di mercato non sono soggetti al rischio di opportunismo delle parti. In secondo luogo, il fenomeno dei distretti è presente in molte parti del mondo, sia avanzato (es. Silicon Valley) che non (paesi emergenti), e molto di quanto accade nei distretti italiani è generalizzabile a questi contesti. Allo stesso modo, l'Italia sarebbe un trampolino interessante per studiare la teoria degli stakeholder o delle imprese familiari, temi di chiaro interesse in realtà diverse dalla nostra e che, per anni, sono stati trascurati dalle analisi più tradizionali.

Spesso si obietta che la nostra tradizione non si fonda o non si presta bene ad essere studiata con le metodologie mainstream nel dibattito internazionale. La questione è importante e rientra nel contesto più ampio della necessità di una pluralità di approcci. Questo tema è sentito a livello internazionale dove si stanno levando molte voci per chiedere maggiore pluralità. Anzitutto, va detto che non c'è tradizione o cultura che si fondi su un metodo piuttosto che un altro. Ogni problema può essere analizzato con diversi strumenti e alcuni aspetti possono essere valorizzati meglio con un approccio (ad esempio quantitativo) mentre altri possono essere valorizzati meglio con un altro approccio (ad esempio qualitativo). Tutto ciò per dire che la tradizione italiana si avvantaggerebbe senz'altro dall'uso degli strumenti analitici della ricerca internazionale mainstream (prevalentemente fondati sull'uso della statistica o di modelli formali). Tuttavia, così come la cultura manageriale italiana deve votarsi di più all'eclettismo metodologico, così deve fare la ricerca internazionale. Come abbiamo detto, queste tendenze sono in atto. Perciò, la soluzione non può e non deve essere che la nostra comunità si chiude per coltivare una nostra pluralità domestica, sterile perché necessariamente ininfluente. Bisogna invece, a partire da quei journal internazionali che non seguono solo il mainstream metodologico, contribuire allo sviluppo di una maggiore varietà di approcci. Del resto sempre più gli editor delle riviste internazionali sono alla ricerca di qualche approccio nuovo, che tuttavia sia solido sotto il profilo metodologico, anche se diverso dall'analisi di tipo meramente statistico. Questo è ad esempio dimostrato dallo sviluppo dei lavori basati non su analisi quantitative ma su esperimenti, o ancora sulle ricerche ad esempio di tipo psicologico e biologico sul tema dei processi decisionali a livello di singolo individuo (dette microfoundations).

\subsection{II riferimento al territorio è importante e non va trascurato?}

Quest'affermazione è del tutto condivisibile. Tuttavia ciò non va inteso nel senso che la ricerca scientifica ha come destinatario il territorio, bensì che quest'ultimo è una fonte importante di dati e di evidenze per una parte delle ricerche, con quei connotati di originalità e generalizzabilità discussi in precedenza. Gli unici destinatari della ricerca scientifica sono i membri della comunità accademica, mentre nella ricerca applicata vi sono, come abbiamo detto, una molteplicità di destinatari, tra cui anche ovviamente il territorio. Ciò non toglie che sia importante, nell'ambito delle attività di servizio, svolgere anche un ruolo importante, per conto dell'università a vantaggio del luogo in cui si opera. 


\subsection{La vicinanza ai temi delle imprese e la rilevanza per i manager sono importanti, per cui è necessario pubblicare sulle riviste accademiche nazionali?}

Quest'affermazione non è a nostro avviso condivisibile. I manager non leggono le riviste accademiche, né internazionali né tantomeno italiane. I manager non leggono neanche le monografie scientifiche, ma dedicano il poco tempo a disposizione per l'aggiornamento ai corsi di formazione o al massimo alla lettura di alcune riviste o di manuali o di opere a taglio divulgativo.

L'opera di divulgazione non è quella della ricerca.

Diverso il discorso sulla creazione di ecosistemi della ricerca, con la presenza congiunta di manager e ricercatori, ma ciò non ha nulla a che vedere con il tipo di pubblicazione.

\subsection{La pubblicazione in libri è importante, perché consente lo sviluppo di un discorso lungo e approfondito, che le riviste non consentono?}

Siamo d'accordo con quest'affermazione. Tuttavia la scrittura di libri va dedicata alla sistematizzazione di un lungo percorso di ricerca o alla divulgazione più che alla redazione di rapporti di ricerca.

In casi rari, può darsi che un articolo non sia appropriato per sviluppare un discorso teorico ampio. Si tratta spesso dell'enunciazione di un nuovo paradigma teorico, che è difficile confinare nello spazio di un articolo (ad es. Nelson e Winter nella piena esplicitazione dell'approccio evolutivo). Ma si tratta di casi veramente rari, che tuttavia sono possibili.

\section{La didattica}

Svolgere un ruolo pedagogico e formativo nello sviluppo dei futuri attori della vita delle imprese e delle organizzazioni.

Tale attività è fondamentale per il potenziamento e la maturazione di quattro livelli formativi: le conoscenze, le attitudini, i valori, i comportamenti.

\subsection{Le conoscenze}

Si tratta della base dell'attività del docente, che deve essere in primis orientata allo sviluppo delle conoscenze sulla gestione delle imprese. Le conoscenze sono soprattutto di linguaggio e di riferimento teorico generale. D'altra parte, è proprio nel riferimento alla conoscenza, che il legame tra didattica e ricerca assume un peso importante. Il trasferimento di conoscenze richiede che si conosca sia la ricerca, anche di frontiera, che la realtà. La difficoltà sta nel coniugare questi due ambiti.

In molti casi, e specie nelle nostre materie, essere un ricercatore, anche di grande respiro, ma incapace di trasmettere il senso di come la ricerca, le sue teorie e le sue 
evidenze empiriche aiutino a comprendere le imprese e più in generale la realtà, è un limite serio.

Altrettanto importante è il limite del docente che, pur conoscendo bene una o più realtà, è in grado di spiegarle solo in termini descrittivi e confinati agli esempi particolari, senza offrire generalizzazioni e la comprensione di dove e come le sue conoscenze si possano applicare ai molti contesti in cui gli attuali discenti, e futuri professionisti, opereranno. D'altra parte, è proprio per questo motivo che è fondamentale la conoscenza approfondita degli sviluppi scientifici delle discipline manageriali . E' il modo con cui si riesce a collocare i fenomeni in contesti più ampi, facendo generalizzazioni e collegamenti, e offrendo ai discenti veri e propri stock di conoscenze e non solo informazioni specifiche relative ad un contesto particolare. Questo è spesso il limite del docente che non fa ricerca: chi è attivo nella comunità scientifica conosce e comprende meglio la ricerca degli altri. Perciò, fare ricerca svolge una funzione importante per la didattica, a tutti i livelli (undergraduate, master, $\mathrm{PhD}$, executive).

Come abbiamo detto all'inizio, il problema è meno rilevante per alcuni corsi di base, dove la diffusione delle conoscenze consente una buona divisione del lavoro tra chi ha qualità e voglia di insegnare bene e chi è relativamente più interessato alla ricerca. Sempre come dicevamo all'inizio, la distinzione deve essere però chiara. Se un Ateneo, un Dipartimento, o un singolo docente, rinuncia alla ricerca, non può proporsi per essere un soggetto alla frontiera nella didattica nei corsi più avanzati (es. master $\mathrm{o} \mathrm{PhD}$ ). Va motivato in base alle performance nella docenza più codificata, mentre chi fa la scelta di operare nel mondo della ricerca va giudicato, incentivato o penalizzato in base a criteri che tengano conto del fatto che ha optato di cimentarsi con un impegno più sfidante.

\subsection{L'atteggiamento}

Le conoscenze non sono sufficienti, cominciando il vero apprendimento quando lo studente svolge un ruolo attivo nel processo. L'atteggiamento attivo fa sì che il soggetto del processo non sia il docente ma lo studente, che se è in grado di svolgere un ruolo attivo innesca un processo di appropriazione e di manipolazione della conoscenza che è la vera base dell'apprendimento: la conoscenza non si acquisisce, ma si produce.

\subsection{I valori}

Compito fondamentale del docente è mettere lo studente di fronte alle responsabilità che un futuro gestore di uomini e risorse ha nei confronti delle persone e della collettività. Si tratta non solo di illustrare il tema dei valori, che pure è importante, ma della necessità di sviluppare un forte senso etico, attraverso l'esempio e il continuo riferimento ai valori della legalità, della responsabilità sociale, del rispetto delle persone, della solidarietà e dell'impegno verso la comunità. A partire dal rispetto delle regole dell'università e dell'aula. 


\subsection{II comportamento}

Il vero obiettivo della formazione della futura classe dirigente delle imprese e delle istituzioni è quello di coniugare conoscenze, atteggiamenti, valori, in comportamenti concreti, che andrebbero favoriti, soprattutto nei corsi di laurea magistrale, attraverso attività concrete ed operative, che pongano lo studente di fronte alla necessità di operare la sintesi che si estrinseca nel comportamento manageriale.

\section{II servizio}

Come dicevamo, è difficile definire in cosa consista il servizio, essendo questo guidato dalle necessità delle diverse università.

Tuttavia sicuramente vi sono almeno tre ambiti in cui l'attività di servizio è importante nella vita del docente:

1. gli organi di governo dell'università: coordinamento di insegnamenti, direzione di corsi di laurea, commissioni didattiche, coordinamento di progetti di ricerca, direzione di dipartimento, organi collegiali e commissioni di funzionamento);

2. presenza qualificata in strutture del territorio

3. attività in organi di gestione della vita pubblica a livello nazionale.

Gli ultimi due vanno trattati con molta delicatezza, essendo talvolta molto difficile distinguere quando prevalga l'interesse dell'università e della collettività e quando invece quello personale.

\section{Bibliografia}

GOODWIN T.H., SAUER R.D. (1995), "Life Cyvle Productivity in Academic Research: Evidence from Cumulative Publication Histories of Academic Economists", Southern Economic Journal, vol. 61, n. 1, pp. 728-743.

WALSH J.P. (2011), "Presidential Address. Embracing the Sacred in Our Secular Scholarly World", Academy of Management Review, vol. 36, n. 2, 215-234. 